\title{
Phase Diagrams of the Blume-Emery-Griffiths thin Films
}

\author{
H. Ez-Zahraouy, L. Bahmad, and A. Benyoussef \\ Faculté des Sciences, Département de Physique, \\ Laboratoire de Magnétisme et Physique des Hautes Energies. \\ B.P. 1014, Rabat, Morocco
}

Received on 11 November, 2005

\begin{abstract}
We study the spin-1 Blume-Emery-Griffiths model with bilinear and biquadratic exchange interactions and single-ion crystal field, using the mean field theory. In addition to the four usual phases: The disordered phase $\mathbf{D}$, the ferromagnetic phase $\mathbf{F}$, the staggered quadrupolar phase $\mathbf{S Q}$ and The ferrimagnetic phase $\mathbf{I}$, we found two new phases, in the case of a thin magnetic film, namely: the sublattice (A or B) non magnetic phase NM and the global non magnetic phase G. These phases are studied, for each layer of the film in the temperature-crystal field plane $(T / J, \Delta / J)$ for different film thicknesses. It is found that the ferrimagnetic and sublattice phases are absent for a monolayer film. Whereas, these phases appear for increasing film thicknessses $N \geq 2$. On the other hand, the thermal behaviour of the layer quadrupolar moments $q_{A}, q_{B}$ and layer magnetisations $m_{A}, m_{B}$ are investigated for negative values of the biquadratic coupling and crystal field. It is also shown that for fixed values of the biquadratic coupling, the temperature and the crystal field, each layer of the film can belong to a different phase. To illustrate this situation, an example is given for $d / J=-1.5, \Delta / J=-3.0$ and $T / J=1.3$.
\end{abstract}

Keywords: Two-sublattice BEG model; Thin films; Staggered quadrupolar phase; Phase diagrams; Multicritical behaviour

\section{INTRODUCTION}

The study of ultra-thin magnetic films, with only few atomic layers thick, is recently subject of modern experimental methods such as the molecular beam epithaxy technique. Very little work has been done in the case of the spin-1 model also called the Blume-Emery-Griffiths BEG. This model was originally introduced [1] in order to explain the phase separation and superfluidity in the ${ }^{3} \mathrm{He}-{ }^{4} \mathrm{He}$ mixtures, as well as to describe other multi-component physical systems, such as metamagnets, liquid crystal mixtures, microemulsions, semiconductor allows, ... etc. The critical properties of this model, for positive values of both the bilinear and biquadratic couplings, were established by different approximation techniques [1-4]. The staggered quadrupolar phase, also called staggered quadrupollar phase, was predicted and investigated on the square lattice by means of mean-field approximation (MFA) and by Monte Carlo (MC) simulations [5]. The transition from the staggered quadrupolar phase to the ferromagnetic phase was established on a three-dimensional cubic lattice [6-8]. The global MFA analysis [6] on this lattice showed a number of other remarkable features such as doubly reentrant behaviour and a ferrimagnetic phase which appears between the staggered quadrupolar phase and the ferromagnetic phase. Latter investigations [8-12] mainly confirmed these results. Using exact solution on the Bethe lattice [13] have constructed the full set of phase diagrams for spin-1 Ising model for both positive and negative biquadratic coupling. The diagrams showed doubly-reentrant behaviour, staggered quadrupolar and ferrimagnetic phases, but the phase diagrams change when changing the coordination number. Extending the biquadratic coupling constant to negative values [14], detailed phase diagrams of the bulk BEG model were established.

On the other hand, the usual phases of the BEG model are found in Ref. [15], in the case of negative biquadratic cou- pling, using the cluster variational theory in the pair approximation but only for a bilayer film with five-fold coordination. The temperature dependey on the order parameters of the Blume-Emery-Griffiths model with repulsive biquadratic coupling on a body centered cubic lattice has been established, using the pair approximation and the path probability method with pair distribution [16]. The metastable and unstable branches of the order parameters and phase transitions of these branches have been established. On the other hand, the effect of an external magnetic field on the multicritical behaviour of the antiferromagnetic Blume-Emery-Griffiths model with repulsive biquadratic coupling has been studied [17] using the lowest approximation of the cluster variation method. This study showed the existence of five topologies of the phase diagrams. When applying the linear chain approximation to establish the Blume-Emery-Griffiths phase diagrams in three different planes, it has been shown [18] that the system exhibits two different behaviours: a first and second order transitions depending on the parameter values.

When applying the Monte Carlo (MC) method for the BlumeEmery-Griffiths model at the ferromagnetic-antiquadrupolardisordered phase interface, it is found in Ref. [19] that a bidimensional system does not present any re-entrant behaviour, while a re-entrance occurs in a bulk system. It is also found that the staggered quadrupolar phase begins to be unstable for a bi-dimensional and three three-dimensional systems.

As far as we know, neither the sublattice non magnetic phase, nor the global non magnetic phase were outlined for the Blume-Emery-Griffiths Ising films. Our aim in this paper is to study the BEG spin-1 film with negative values of the biquadratic coupling for different film thicknesses. The phase diagrams we obtained contain some new phases: The sublattice (A or B) non magnetic phase $\mathbf{N M}$ and the global non magnetic phase $\mathbf{G}$. In addition to the usual phases obtained by several authors, namely: the disordered phase $\mathbf{D}$, the ferromagnetic phase $\mathbf{F}$, the staggered quadrupolar phase $\mathbf{S Q}$ and the ferrimagnetic phase $\mathbf{I}$. The paper is organized as follows. 
In the Sect. 2 we define the model and the used method. Resulting phase diagrams are presented and discussed in Sect. 3. The final Sect. 4. is devoted to conclusions.

\section{MODEL AND METHOD}

The Blume-Emery-Griffiths model, with spins $s_{i}=0, \pm 1$, is characterized by two order parameters: magnetization $m$ and quadrupolar moment $q$, defined respectively by:

$$
m=\left\langle s_{i}\right\rangle, \quad q=\left\langle s_{i}^{2}\right\rangle
$$

The case we are studying is a bipartite lattice, i.e. the lattice is divided on two sublattices A and B, such that every site belonging to $\mathrm{A}$ is surrounded only by sites belonging to $\mathrm{B}$ and vice versa. To account the two-sublattice structure we need four order parameters: $m_{A, B}=\left\langle s_{i}\right\rangle_{A, B}$ and $q_{A, B}=\left\langle s_{i}^{2}\right\rangle_{A, B}$, where $\mathrm{A}, \mathrm{B}$ denotes sublattices.

The Hamiltonian describing the model is defined by:

$$
\mathcal{H}=-J \sum_{<i j>} s_{i} s_{j}-d \sum_{<i j>} s_{i}^{2} s_{j}^{2}+\sum \Delta_{i} s_{i}^{2}
$$

where $s_{i}$ takes the values $0, \pm 1$, at each lattice site, $\langle i j\rangle$ denotes a summation over all nearest-neighbour pairs, $J$ and $d$ are, respectively, the correspondingly bilinear and biquadratic interaction couplings. $\Delta_{i}$ is the crystal field applied on each site $^{\prime} i \prime$ of the layer $k$ of the film formed with $N$ layers, so that:

$$
\Delta_{i}=\Delta_{k}=\Delta / k^{\alpha}
$$

In all the following we will be limited to the case $\alpha=1$, and a bilinear coupling value $J=1$.

From the Hamiltonian Eq.(2), the magnetisation and quadrupolar moment of each layer $k$, of the film formed with $N$ layers, are given for each sublattice $A$ (or $B$ ) respectively, by:

$$
m_{k}^{A(B)}=\frac{\operatorname{Tr}\left(s_{k}^{A(B)} \exp \left(-\beta \mathcal{H}_{0}\right)\right)}{\operatorname{Tr}\left(\exp \left(-\beta \mathcal{H}_{0}\right)\right)}
$$

and

$$
q_{k}^{A(B)}=\frac{\operatorname{Tr}\left[s_{k}^{A(B)}\right]^{2} \exp \left(-\beta \mathcal{H}_{0}\right)}{\operatorname{Tr}\left(\exp \left(-\beta \mathcal{H}_{0}\right)\right)},
$$

where the spin variables $s_{k}^{A(B)}$ belong to the layer $k$ ( $k=$ $1, \ldots, N)$ and the sublattice $A$ (resp. $B$ ). In the above equations the trace $T r$ is performed using the global Hamiltonian $\mathcal{H}_{0}$ which is the sum of each layer term $\mathcal{H}_{0 k}$ so that:

$$
\begin{aligned}
& \mathcal{H}_{0 k}=-\left[4 \cdot s_{k}^{B}+s_{k-1}^{B}+s_{k+1}^{B}\right] s_{k}^{A}-\left[4 \cdot s_{k}^{A}+s_{k-1}^{A}+s_{k+1}^{A}\right] s_{k}^{B}-d \cdot\left[4 \cdot\left(s_{k}^{B}\right)^{2}+\left(s_{k-1}^{B}\right)^{2}\right. \\
& \left.+\left(s_{k+1}^{B}\right)^{2}\right]\left(s_{k}^{A}\right)^{2}-d \cdot\left[4 \cdot\left(s_{k}^{A}\right)^{2}+\left(s_{k-1}^{A}\right)^{2}+\left(s_{k+1}^{A}\right)^{2}\right]\left(s_{k}^{B}\right)^{2}+\Delta\left[\left(s_{k}^{A}\right)^{2}+\left(s_{k}^{B}\right)^{2}\right]
\end{aligned}
$$

with the free boundary conditions: $m_{0}^{A(B)}=m_{N+1}^{A(B)}=0$ and $q_{0}^{A(B)}=q_{N+1}^{A(B)}=0$.

Let $Z_{0}=\exp \left(-\beta \mathcal{H}_{0}\right)$ be the partition function of the system; the global free energy is defined as: $\mathcal{F}=\mathcal{F}_{0}+<\mathcal{H}-\mathcal{H}_{0}>_{0}$ with: $\mathcal{F}_{0}=-\frac{1}{\beta} \log \left(Z_{0}\right)$.

The average spin configuration $\langle\ldots\rangle_{0}$ is performed using the global Hamiltonian $\mathcal{H}_{0}$ defined as:

$$
\mathcal{H}_{0}=\sum_{k}^{N} \mathcal{H}_{0 k}
$$

Hence, the global free energy can be written as:

$$
\begin{aligned}
& \mathcal{F}=-\frac{1}{\beta} \log \left(Z_{0}\right)+\sum_{k=1}^{N}\left[\frac{J}{4} m_{k}^{A}\left(4 \cdot m_{k}^{B}+m_{k-1}^{B}+m_{k+1}^{B}\right)+\frac{d}{4} q_{k}^{A}\left(4 \cdot q_{k}^{B}+q_{k-1}^{B}+q_{k+1}^{B}\right)\right. \\
& \left.+\frac{J}{4} m_{k}^{B}\left(4 \cdot m_{k}^{A}+m_{k-1}^{A}+m_{k+1}^{A}\right)+\frac{d}{4} q_{k}^{B}\left(4 \cdot q_{k}^{A}+q_{k-1}^{A}+q_{k+1}^{A}\right)\right]
\end{aligned}
$$

where $\beta=1 / k_{B} T: T$ stands for the absolute temperature and $k_{B}$ the Boltzmann constant.

The equations (4) and (5) are solved numerically using the iterative method. Different initial guesses can lead to different solutions; the one which makes the free energy smallest is selected as the global minimum. 
The parameters defining the different phases of the BEG model are:

1. The ferromagnetic phase $\mathbf{F}$ :

2. The ferrimagnetic phase I :

$$
\begin{array}{lll}
\text { 1. The ferromagnetic phase } \mathbf{F}: & m_{A}=m_{B} \neq 0, & q_{A}=q_{B} \\
\text { 2. The ferrimagnetic phase } \mathbf{I}: & 0 \neq m_{A} \neq m_{B} \neq 0, & q_{A} \neq q_{B} \\
\text { 3. The staggered quadrupolar phase SQ : } & m_{A}=m_{B}=0, & q_{A} \neq q_{B}
\end{array}
$$$$
\text { 4. The disordered phase } \mathbf{D} \text { : } \quad m_{A}=m_{B}=0, \quad q_{A}=q_{B} \neq 0
$$

5. The global non magnetic phase $\mathbf{G}: m_{A}=0 \quad m_{B}=0, q_{A}=0 \quad q_{B}=0$

6. The sublattice (A or B) non magnetic phase $\mathbf{N M}$ is defined either

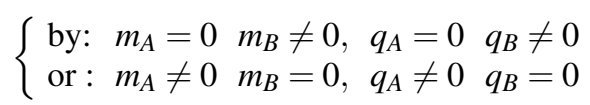

\section{RESULTS AND DISCUSSIONS}

In order to investigate the existing phases of a BEG monolayer film we plot in Fig. 1 the corresponding phase diagram in the plane $(T / J, \Delta / J)$. This diagram shows that the disordered phase $\mathbf{D}$ is reached at higher temperatures terminating respectively, the ferromagnetic phase $\mathbf{F}$ for negative and large values of the crystal field $\Delta / J$, the staggered quadrupolar SQ phase for $\Delta / J \approx 0$ and the $\mathbf{G}$ phase for $\Delta / J \geq 1$. In addition, the disordered phase $\mathbf{D}$ appears also at very low temperatures for positive values of the crystal field $\Delta / J \approx 0.5$ while the phase SQ replaces the phase $\mathbf{N M}$ at very low temperature values. It worth to note that the phases I and NM are absent for a monolayer BEG film. Hence we investigate the phase evolutions in a BEG bilayer film. As it is shown in Fig. 2a corresponding to the first layer $k=1$, and a biquadratic coupling constant value $d / J=-1.5$. The phase diagram in the plane $(T / J, \Delta / J)$, shows that the ferrimagnetic phase $\mathbf{I}$ is absent for a monolayer film. This phase is found only for low temperatures between the phases $\mathbf{F}$ and $\mathbf{S Q}$. The other phases, $\mathbf{D}$ and $\mathbf{G}$, found for a monolayer film are still present. The phase $\mathbf{D}$ appears for $T / J \geq 0.5$ and disappears for lower temperatures where the phase $\mathbf{G}$ takes place for positive values of the crystal field. In addition the phase SQ replaces the phase NM existing at very low temperatures. The disordered phase D appears also at very low temperatures for positive values of the crystal field when $\Delta / J \approx 0.5$. Concerning a deeper layer $k=2$ of this bilayer film, Fig. 2 b, we found that in comparison with the first layer, there is two important regions: (i) The phase $\mathbf{I}$ is still present and (ii) the phase $\mathbf{D}$ is found even for $T / J \leq 0.5$ provided that the crystal field is kept with positive values. The other phases: F, SQ and $\mathbf{G}$ are also present for this layer.

In order to envisage the film thickness effect on different phase, we consider a thin film formed with $N=5$ layers. Keeping the biquadratic coupling at a constant and negative value $d / J=-1.5$ we study the behaviour of each layer under the temperature and crystal field effects. The phase dia-

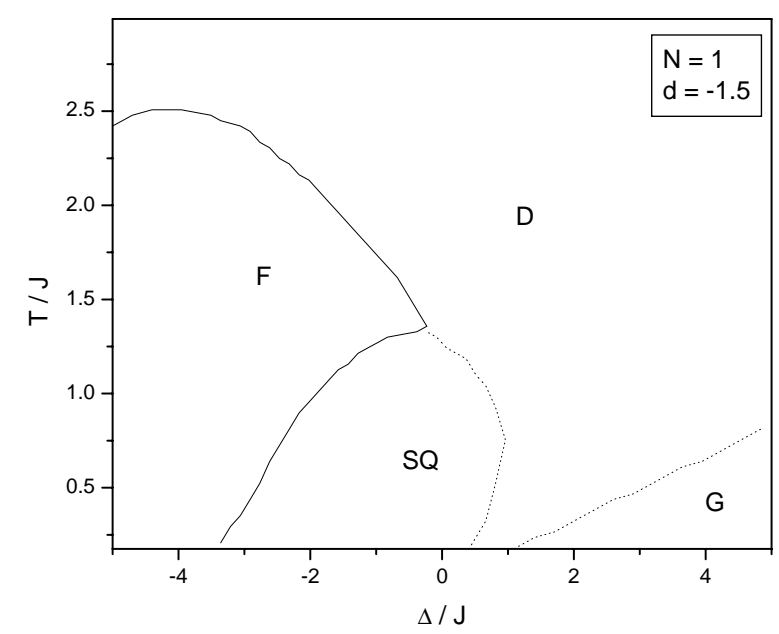

FIG. 1: Phase diagram of the BEG monolayer film $N=1$ in $(T / J, \Delta / J)$ plane for $d / J=-1.5$. The phase $\mathbf{D}$ is reached at higher temperatures surrounding the phases $\mathbf{F}$ and $\mathbf{G}$ when increasing temperature values. The phases: ferrimagnetic I and sublattice non magnetic NM are absent for a monolayer. Solid lines are first order transitions, whereas dashed lines are second order transitions.

gram of the first layer, plotted in $(T / J, \Delta / J)$ plane, see Fig. 3a, shows that the ferrimagnetic phase $\mathbf{I}$ is reached at higher temperatures $T / J \approx 1.5$ and the phase $\mathbf{G}$ emerges rapidly even for small and positive values of the crystal field $\Delta / J$. For a deeper layer $k=3$, see Fig. 3b, the phase NM takes place between the phases $\mathbf{I}$ and $\mathbf{S Q}$ at low temperatures. While the phase $\mathbf{I}$ is still present and the phase $\mathbf{G}$ region decreases in comparison with that one of the first layer. Concerning the other deeper layers, the phase I and the phase NM persist and some regions of the phase $\mathbf{I}$ are detached from each other leaving place to the phase NM. We found that not only the phase $\mathbf{G}$ region decreases more and more when the order of the layer increases, but also the region of this phase decreases when increasing 
(a)

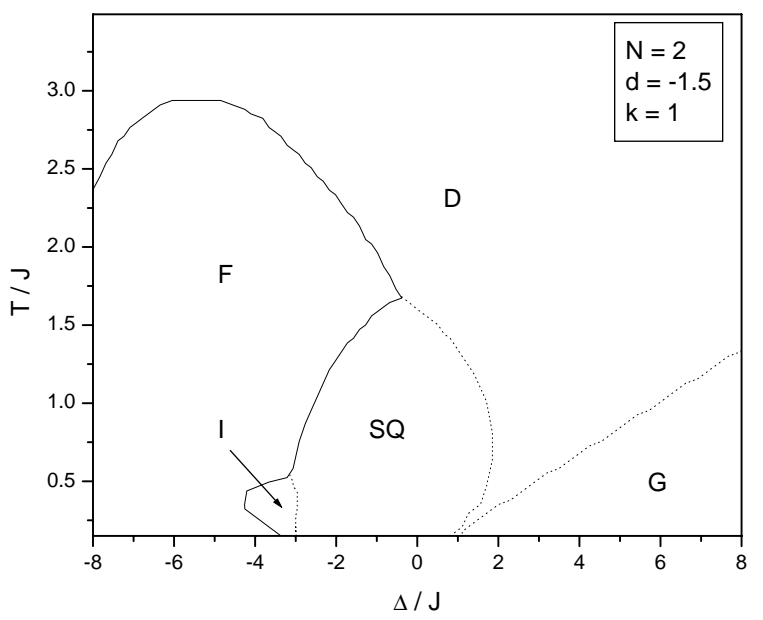

(b)

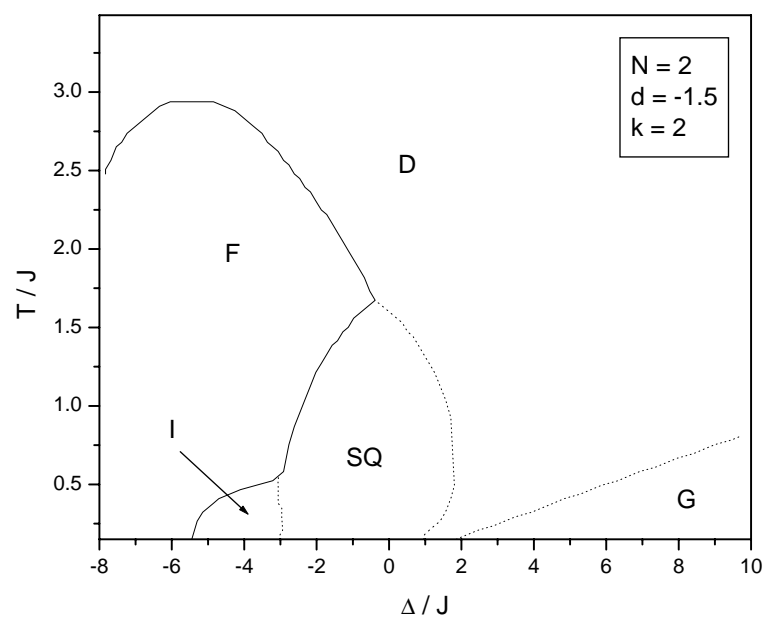

FIG. 2: Phase diagrams of a BEG bilayer film $N=2$ for $d / J=-1.5$ in $(T / J, \Delta / J)$ plane: (a) For the first layer $\mathrm{k}=1$ : the ferrimagnetic phase $\mathbf{I}$ appears for low temperatures between the phases $\mathbf{F}$ and $\mathbf{S Q}$. It is worth to note that the phases: $\mathbf{D}$ and $\mathbf{G}$, found for a monolayer film, are still present for the bilayer film case. (b) For the second layer $k=2$ one can note two important points: (i) the region of the ferrimagnetic phase $\mathbf{I}$ is greater than that one of the first layer and (ii) the phase $\mathbf{D}$ is found even for $T / J \leq 0.5$ provided that the crystal field is kept with positive values. The other phases: F, SQ and $\mathbf{G}$ are still present for this layer.

the layer order. Moreover the phase D appears even at low temperatures for the last layer $k=N$ of the film.

To complete this study, we investigate the thermal dependence of the magnetisations $m_{A}, m_{B}$ and quadrupolar moments $q_{A}, q_{B}$ for a BEG thin film formed with $N=5$ layers, for $d / J=-1.5$ and $\Delta / J=-3.0$. Indeed, we found that concerning the first layer, see Fig. 4a, the three phases I, SQ and D shown in Fig. 3a when $\Delta / J=-3.0$, are well illustrated in this figure, when increasing temperatures. The phase $\mathbf{I}$ found at very low temperature is followed by the phase $\mathbf{F}$ and the phase D is reached at high temperatures. Regarding a deeper layer, (a)

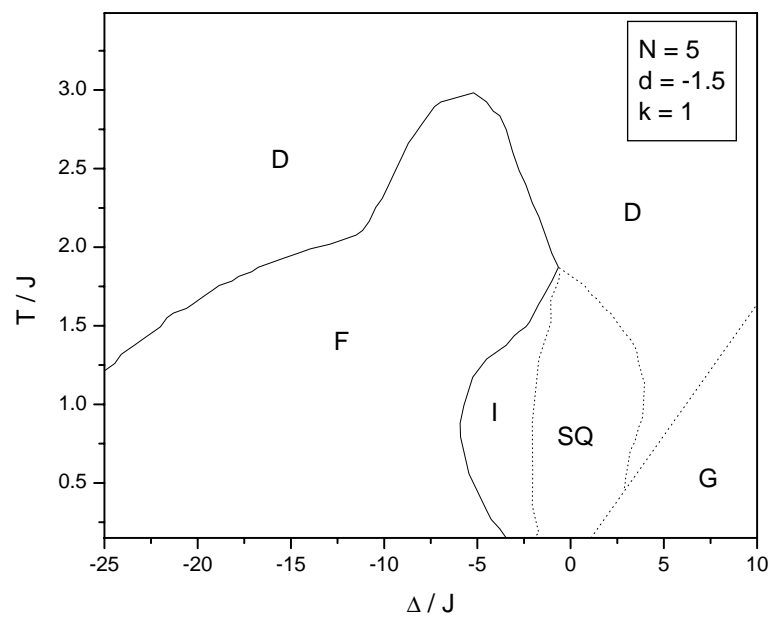

(b)

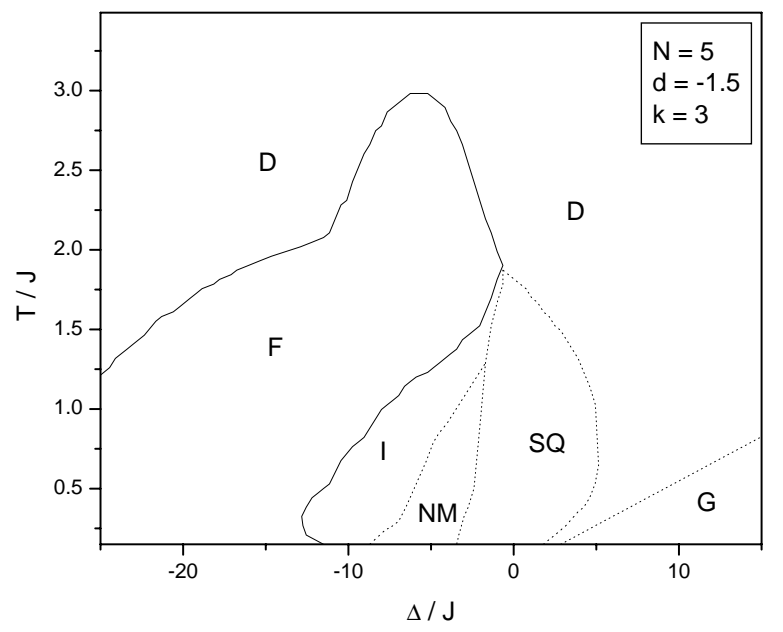

FIG. 3: Phase diagrams of a BEG thin film with $N=5$ layers for $d / J=-1.5$ in $(T / J, \Delta / J)$ plane: (a) The properties of the bilayer film still exist for the first layer $k=1$ of this thin film, but its phase diagram shows that not only the ferrimagnetic phase $\mathbf{I}$ is reached at higher temperatures $T / J \approx 1.5$ but also that the phase $\mathbf{G}$ emerges rapidly even for small and positive values of the crystal field $\Delta / J$. (b) For a deeper layers, $k=3$ for example, the phases: I and NM persist and some regions of the phase $\mathbf{I}$ are detached from each other leaving the place to the phase NM. The phase $\mathbf{G}$ is found for positive and increasing values of the crystal field $\Delta / J$ and low temperature values.

for example $k=3$ see Fig. 4b, we found that the phase NM appears at low temperature followed by the phase $\mathbf{I}$, then the phases $\mathbf{F}$ and $\mathbf{D}$ are reached, respectively. The phase NM persists for deeper layers, whereas the phase I region decreases and terminates by the phase D. For each layer of the film, the usual phases: F, SQ, D and $\mathbf{I}$ are present in addition to the new phases, for specific values of temperature $T / J$, biquadratic coupling $d / J$ and crystal field $\Delta / J$. 


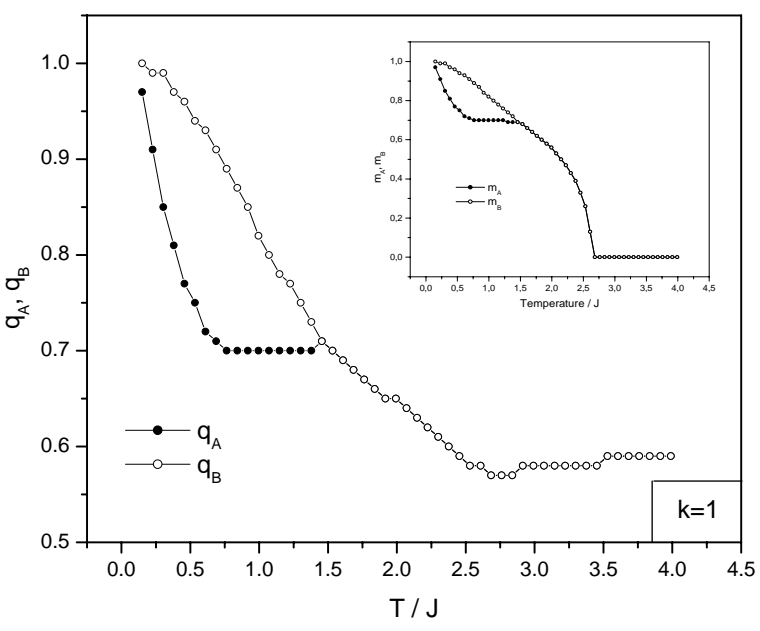

a)

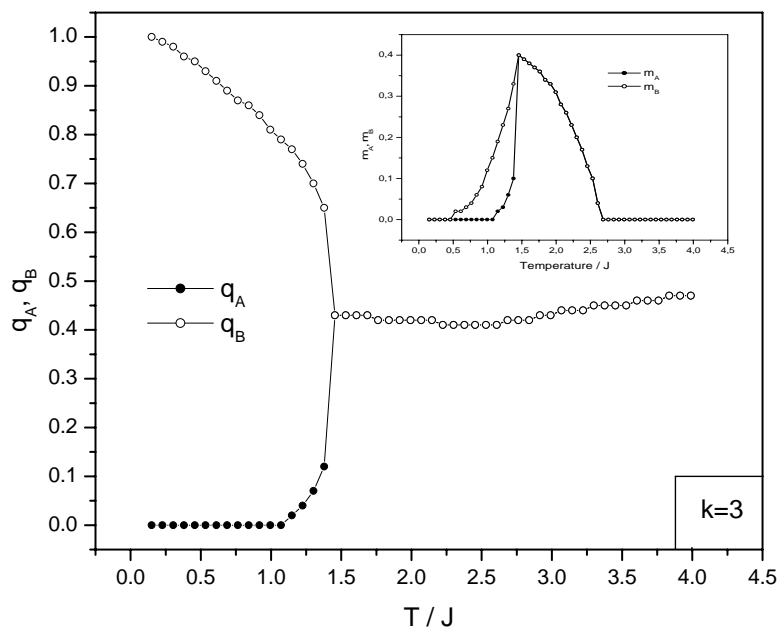

b)

FIG. 4: Thermal dependence of the magnetisations $m_{A}, m_{B}$ and quadrupolar moments $q_{A}, q_{B}$ for a BEG thin film with $N=5$ layers, $d / J=-1.5$ and $\Delta / J=-3.0$. (a) For the first layer $k=1$, the three phases: I, SQ and $\mathbf{D}$ shown in Fig. 3a, are well illustrated in this figure, for increasing temperatures. The phase $\mathbf{I}$ found at very low temperature is followed by the phase $\mathbf{F}$ and the phase $\mathbf{D}$ is reached at high temperatures. (b) For a deeper layer $k=3$, the phase $\mathbf{N M}$ appears at a very low temperature followed by the phase $\mathbf{I}$, then the phases: $\mathbf{F}$ and $\mathbf{D}$ are reached, respectively.

\section{CONCLUSION}

In this work we have studied the phase evolutions in the BEG spin-1 film with biquadratic exchange interactions and single-ion crystal field, using the mean field theory. We showed that in addition to the four usual phases: disordered $\mathbf{D}$, ferromagnetic $\mathbf{F}$, staggered quadrupolar $\mathbf{S} \mathbf{Q}$ and ferrimagnetic I, some new phases are present in the case of a thin magnetic film, namely: the sublattice A (or B) non magnetic phase NM and the global non magnetic phase $\mathbf{G}$. All these phases are studied for a monolayer, bilayer and thin film, for each layer of the film in the temperature-crystal field plane. Moreover, the thermal behaviour of each layer quadrupolar moments $q_{A}, q_{B}$ and magnetisations $m_{A}, m_{B}$ are investigated for fixed values of the biquadratic coupling and crystal field. It is also shown that for fixed values of the biquadratic coupling $d / J$, temperature $T / J$ and the crystal field $\Delta / J$, the different layers of the film exhibit different phases.
[1] M. Blume, V. J. Emery, and R. B. Griffiths, Phys. Rev. A 4, 1071 (1971)

[2] D. Mukamel and M. Blume, Phys. Rev. A 10, 619 (1974).

[3] D. Furman, S. Dattagupta, and R. B. Griffiths, Phys. Rev. B 15, 441 (1977).

[4] A. N. Berker and M. Wortis, Phys. Rev. B 14, 4946 (1976).

[5] M. Tanaka and T. Kawabe, J. Phys. Soc. Jpn. 54, 2194, (1985).

[6] W. Hoston and A. N. Berker, Phys. Rev. Lett. 67, 1027 (1991).

[7] W. Hoston and A. N. Berker, J. Appl. Phys. 70, 6101 (1991).

[8] K. Kasono and I. Ono, Z. Phys. B 88, 205 (1992).

[9] R. R. Netz, Europhys. Lett. 17 (4), 373 (1992).

[10] R.R. Netz and A. N. Berker, Phys. Rev B 47, 15019 (1993).

[11] A. Rosengren and S. Lapinskas, Phys. Rev. Lett 71, 165 (1993).

[12] S. Lapinskas and A. Rosengren, Phys. Rev. B 49, 15190 (1994).
[13] A. Z. Akheyan and N. S. Ananikian, J. Phys. A 25, 3111 (1992).

[14] W. Hoston and A. Nihat Berker, Pyhs. Rev. Lett. 67, 1027 (1992).

[15] J. W. Tucker, T. Balcerzak, M. Gzik, and A. Sukienniki, J. Magn. Magn. Mater. A 187, 381 (1998).

[16] M. Keskin and A. Erdin , J. Magn. Magn. Mater. 283, 392 (2004).

[17] A. Erdin, O. Canko, and M. Keskin, J. Magn. Magn. Mater. in press (2005).

[18] E. Albayrak and M. Keskin, J. Magn. Magn. Mater. 203, 201 (2000).

[19] A. Rachadi and A. Benyoussef, Phys. Rev. B 69, 64423 (2004). 